\title{
DOES THE SYSTEM THETA VIRGINIS REPRESENT A NEW GLASS OF PULSATING STAR?
}

\author{
W.R. Beardsley and E.R. Zizka \\ Allegheny Observatory \\ University of Pittsburgh
}

\begin{abstract}
RESUMEN
El análisis de la variación de la velocidad radial de $\theta$ Virginis $\mathrm{A}$ ha revelado que superpuesta a la variación orbital de 17 años de período, existe un período de variación ultracorto de 0.15 días. Este ciclo secundario se atribuye a pulsaciones. Se discute el tipo de estrella pulstante que $\theta$ Virginis A puede ser.
\end{abstract}

\begin{abstract}
Analysis of the radial velocity variation of $\theta$ Virginis $A$ has revealed that, superposed on an orbital variation with a period of 17 years, there is present an ultrashort periodic variation of 0.15 -day. This secondary cycle is attributed to pulsation. The question of what type of pulsating star $\theta$ Virginis A may represent is discussed.
\end{abstract}

\section{INTRODUCTION}

The star $\theta$ Virginis [ADS 8801A; $\alpha=13^{\mathrm{b}} 04^{\mathrm{m}} \cdot 7$, $\left.\delta=5^{\circ} 00^{\prime}(1900), m_{r}=4.37, A 1 \mathrm{~V}\right]$ was announced by Mitchell (1909) as having variable radial velocity. Subsequent observations, primarily at Lick and Yerkes observatories, were summarized by Frost $e t$ al. (1929) who stated in reference to the series of Yerkes velocities: "The gradual change from positive to negative values is very clearly indicated and also substantiated by Lick velocities." An examination of these velocities by one of the authors suggested that while the gradual change was indeed evident, a surprisingly large velocity scatter existed despite the sharp-lines in the spectrum, suggesting a short period variation and the possible presence of a third body in the system. Accordingly, in 1962 the star was placed on the radial velocity program at the Allegheny Observatory.

Conti (1955) and Deutsch (1967)' have called attention to the existence of an excess number of stars having relatively small rotational velocities, (Vsin i), among early A-type stars. In a further investigation Conti and Strom (1968) included $\theta$ Virginis as one of four extremely sharp-lined field stars in their investigation as to whether this excess group possessed abundance anomalies similar to those found for Am stars. They concluded that element abundances in $\theta$ Virginis were normal. A photoelectric spectrophotometric study of standard stars by Oke (1964) also implies that $\theta$ Virginis is quite normal in monochromatic flux throughout the blue-green region. In addition 5-color photometry (Iriarte et al. 1965) implies normal color indices in the range $\mathrm{U}$ to $\mathrm{I}$.

\section{OBSERVATIONAL DATA}

In addition to 53 published early radial velocities (Abt and Biggs 1972), a total of 110 observations were obtained at the Allegheny Observatory during the interval 1962-1974 followed by three observations at Kitt Peak National Observatory in 1975. These observations do not quite span one full orbital period, hence the period determined in this investigation 
depends strongly on the 1975 velocities and must still be considered as provisional. The sharp lines in the spectrum of this star allow the Allegheny spectra $\left(40 \AA \mathrm{mm}^{-1}\right)$ to be measured with an average plate accuracy of $1 \mathrm{~km} \mathrm{~s}^{-1}$ using a Grant oscilloscope display measuring engine. A full listing of the observed radial velocities will be published separately.

\section{THE SHORT PERIOD VELOCITY VARIATION}

The aforementioned presence of scatter in the older radial velocities was found to be present also in the Allegheny observations. Consequently initial efforts to determine the long period orbit were based on seasonal mean velocities. As this long orbit became better established, efforts were begun to define the possible short period variation. For a considerable time these efforts were not successful. Finally, noting that Vega has a characteristic sharp lined spectrum similar to that of $\theta$ Virginis, it was decided to search for an ultrashort periodicity such as Vega is known to possess (Beardsley 1969). This search was soon successful and a period of $0.152360 \pm$ 0.000001 day was ultimately derived; the variation having a semi-amplitude of $5 \mathrm{~km} \mathrm{~s}^{-1}$. The phase

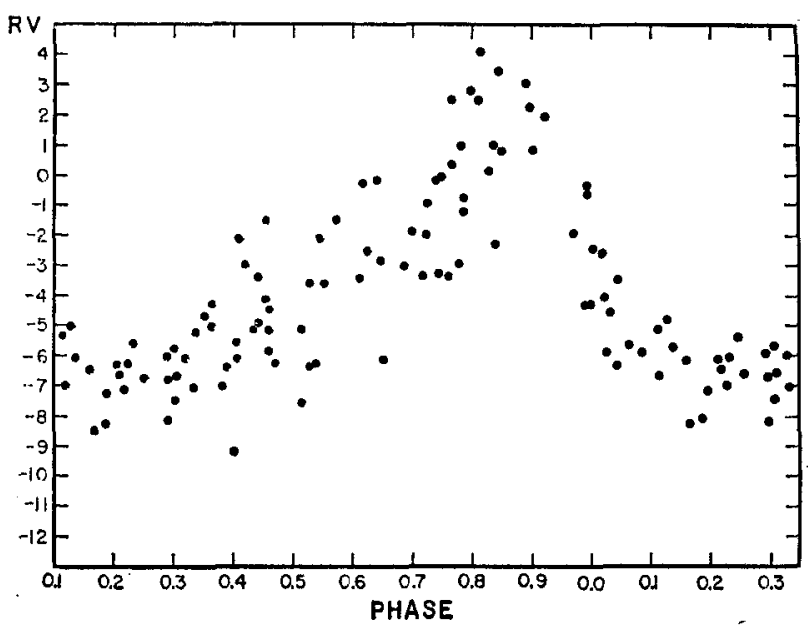

Fig. 1. A plot of the short period residuals from the long period orbit normalized to the orbital systemic velocity. This plot represents the interval 1962-1975. The period is $0.152360 \pm 0.000001$ day. This variation is attributed to pulsation. The ordinate-axis represents $\mathrm{km} \mathrm{s}^{-1}$. is counted from $T_{0}=J D 2438110.8406$ when our initial peak velocity occurred. Plotting the observations of each season using this period permitted the determination of seasonal systemic velocities which were then used to derive the long period orbit. In Figure 1 we plot the short period residuals from the long period orbit normalized to the orbital systemic velocity. This plot represents the interval 1962-1975. The magnitude of the scatter is nearly what one might expect for a mean standard error of measurement of about $\pm 1 \mathrm{~km} \mathrm{~s}^{-1}$.

The authors are of the opinion that the extremely short period (3.6 hours) invalidates the older observations, due to their generally long exposure times, in regard to both the short period variation and the seasonal mean velocities in the long period orbit. It is worth noting however, that two 1906 Lick velocities and several 1907 Yerkes velocities are systematically too positive, indicating that perhaps at that time the amplitude of the short period variation may have been considerably greater than that indicated by the present series.

\section{THE SPEGTROSCOPIC ORBIT}

The short period variation in radial velocity having been determined, it was removed from the individual observations. The adjusted velocities were then used to do an orbital solution for differential corrections to the preliminary seasonal mean orbital values. The final orbital elements are:

$$
\begin{aligned}
\mathrm{P} & =17.84 \pm 0.05 \text { years } \\
\mathrm{T} & =1969.776 \pm 0.83 \\
\omega & =30198 \pm 1496 \\
\mathrm{e} & =0.33 \pm 0.24 \\
\mathrm{~K} & =3.18 \pm 0.04 \mathrm{~km} / \mathrm{sec} \\
\mathrm{Vo} & =-4.19 \pm 0.45 \mathrm{~km} / \mathrm{sec} \\
\text { a } \sin \mathrm{i} & =268.9 \times 10^{6} \pm 64.8 \times 10^{6} \mathrm{~km} \\
\mathrm{f}(\mathrm{m}) & =0.018
\end{aligned}
$$

A plot of this orbit is shown in Figure 2. Each point represents a seasonal mean orbital velocity as calculated from the orbit. The error bars represent the observational scatter which appeared in Figure 1, now projected onto the spectroscopic orbit. One might expect that this orbit is also detectable 


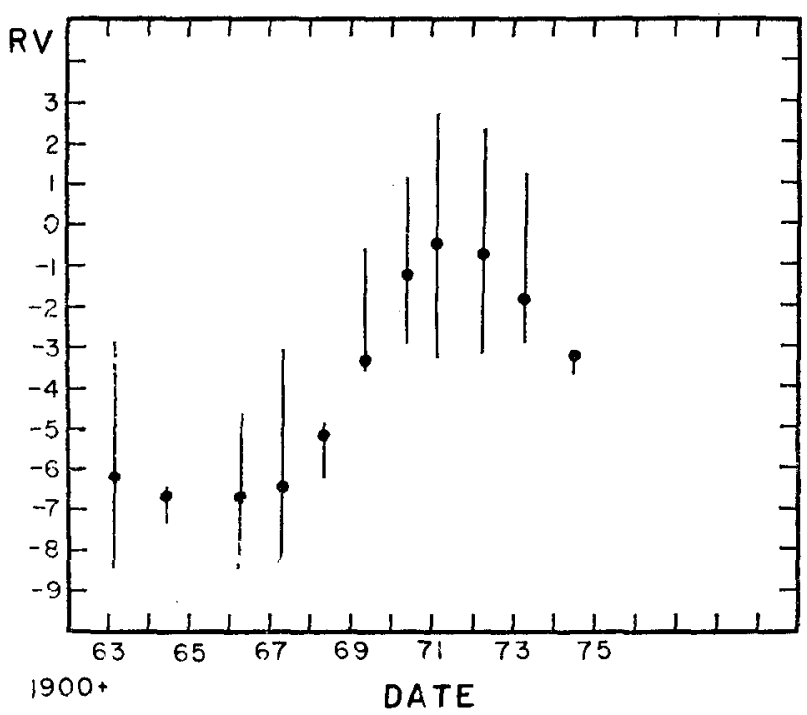

Fig. 2. A plot of seasonal mean orbital velocity during the interval $1962-1975$ as calculated from the 17.84-year orbit. The error bars represent the observational scatter which appeared in Figure 1, now projected onto the spectroscopic orbit. The ordinate-axis represents $\mathrm{km} \mathrm{s}^{-1}$.

astrometrically. Unfortunately, the available astrometric observations at Allegheny Observatory are presently inadequate for this purpose.

\section{DISGUSSION}

We are left now with the question of the nature of the short period variation in radial velocity. If Vega is a similar star, as postulated earlier, then the two stars might be expected to have similar radii. For the case of Vega the radius has been measured interferometrically to be about $3.2 \mathrm{R}_{\odot}$ (Brown et ai. 1964). Assuming that $\theta$ Virginis has a mass of 3.5 $\mathrm{M}_{\odot}$ in accordance with normal early A-type stars and that the variation in velocity is due to orbital motion, then the calculated semi-major axis for the orbit is only $1.6 \mathrm{R}_{\odot}$. This is much too small a value to allow orbital motion to be considered as a plausible explanation for the velocity variation. Rejecting orbital motion leaves us with pulsation as the apparent cause. Support for this hypothesis is evident in the radial velocity curve. A possible standstill is seen at the mid-point of the slow rise to maximum positive velocity and it occurs, as one would expect, at the maximum radius of the star. The star, however, does not fit the period-density relationship for cepheids, which may be written in the form:

$$
\mathbf{P}=0.022\left(\frac{\mathrm{M}^{0.7}}{\mathbf{R}^{1.7}}\right)
$$

If one solves for the mass using the aforementioned radius, the result is only $0.9 \mathrm{M}_{\odot}$ entirely too small a value for an early A-type star. A pulsation constant of 0.05 would, however, bring period mass and radius into agreement.

As previously mentioned we suspect Vega is a similar pulsating star. We suspect that $\gamma$ Geminorum also pulsates with an ultrashort period, since it too shows an unreasonable amount of scatter in radial velocity throughout a 12.4 -year orbit. The locations of all three of these stars on the $H-R$ diagram are in close proximity to that of the star $\gamma$ Ursa Minoris, which Struve (1955) suggested may define a pulsation sequence with the star Maia. This Maia sequence, as Struve referred to it, has since remained hypothetical and unestablished. Is it now reasonable to suppose that $\theta$ Virginis represents a new member of this Maia sequence? Or might $\theta$ Virginis be an extension of the $\delta$ Scuti class of variable stars? Baglin et al. (1973) describe such stars as having spectral types between $\mathrm{A} 2$ and F5 with luminosity classes $V$ to II and mostly normal spectra. It would seem that a decision in this matter must await studies of additional late $\mathrm{B}$ and early A-type stars.

The authors express their appreciation to the National Science Foundation which helped to support the observations.

\section{REFERENCES}

Abt, H. A., and Biggs, E. S. 1972, Bibliography of Stellar Radial Velocities, (Tucson: Kitt Peak National Obs.). Baglin, A., Breger, M., Chevalier, C., Hauck, B., le Contel, J. M., Sareyan, J. P., and Valtier, J. C. 1973, Astron. and Astrophys., 23, 221.

Beardsley, W. R. 1969, Pub. Allegheny Obs., 8, No. 7.

Brown, R. H., Hazard, C., Davis, J., and Allen, L. R. 1964, Nature, 201, 1111.

Conti, P. S. 1965, Ap. J., 142, 1594.

Conti, P. S., and Strom, S. E. 1968, Ap. J., 154, 975.

Deutsch, A. J. 1967, in The Magnetic and Related Stars, ed. R. C. Cameron (Baltimore: Mono Book Corp.). 
Frost, E. B., Barrett, S. B., and Struve, O. 1929, Pub. Mitchell, S. A. 1909, Ap. J., 30, 239.

Yerkes Obs., 7, Part 1, 45.

Mitchell, S. A. $1909, A p ., 30,239$
Oke, J. B. $1964, A p$. J., $140,689$.

Iriarte, B., Johnson, H. L., Mitchell, R. I., and Wisniewski, $\quad$ Struve, O. 1955, Sky Telesc., 14, 461.

W. K. 1965, Sky Telesc., 30, 21.

\section{DISCUSSION}

$A b t$ : This sounds as though it is a member of Struve's Maia sequence, which are late B stars. Most late B stars have lines too broad to detect so small a velocity variation. 